\title{
Acute respiratory failure following scorpion stings: Anaphylaxis or severe systemic envenomation?
}

\author{
Austin Castillo BS, Pradeep Attaluri BS
}

\begin{abstract}
Scorpion stings can cause a range of reactions which includes severe systemic envenomation resulting in respiratory failure, cardiovascular collapse, and severe neurologic complications. Patients with allergies to scorpion venom or allergies to bee and ant venom can have anaphylactic reactions following scorpion stings. We report a patient who developed severe respiratory distress following 2-3 scorpion stings on her face. She required an emergency cricothyroidotomy and prolonged hospitalization, related in part to underlying comorbidity. Physicians caring for patients following scorpion stings need to remember that these patients can have severe reactions to the venom, including anaphylactic reactions. The initial management likely involves respiratory and cardiac resuscitation. These patients may have a very difficult intubation and require an emergency surgical airway.
\end{abstract}

Keywords: scorpion, envenomation, acute respiratory failure, anaphylaxis

\section{INTRODUCTION}

Scorpions (e.g., Centruroides vittatus, the striped bark scorpion) are found throughout the United States, especially in the Southwest where the climate is dry and warm (Figure). Scorpion stings are generally considered non-life threatening. The National Poison Data System documented 15,604 reported stings in $2015 ; 1,416$ required treatment at a healthcare facility. Outcomes were classified as none: 76 , minor: 10,040, moderate: 698 , major: 25 , and death: $0 .^{1}$

\section{CASE}

A 58-year-old woman presented to her local emergency department complaining of difficulty breathing; her husband told the physician that she was stung 2-3 times by a scorpion when she put on her BiPAP mask. She took diphenhydramine at home, but this

Corresponding author: Austin Castillo

Contact Information: Austin.c.castillo@ttuhsc.edu DOI: $10.12746 /$ swrccc.v6i22.435 did not relieve her symptoms. She had labored breathing with retractions and was making grunting noises. She also had a swollen tongue and throat, stridor, and diminished breath sounds; she had no skin rash. Vital signs included blood pressure 160/93 $\mathrm{mmHg}$, heart rate 95 beats per minute, and respiratory rate 22 breaths per minute. Initial laboratory testing showed WBC $10.2 \mathrm{k} / \mu \mathrm{L}$ with $2.1 \%$ eosinophils, hemoglobin $13.4 \mathrm{gm} / \mathrm{dL}$, and platelet count $284 \mathrm{k} / \mu \mathrm{L}$. A complete chemistry panel results included sodium $140 \mathrm{mEq} / \mathrm{L}$, potassium $3.6 \mathrm{mEq} / \mathrm{L}$, chloride $104 \mathrm{mEq} / \mathrm{L}$, BUN 15 $\mathrm{mg} / \mathrm{dL}$, and creatinine $1.0 \mathrm{mg} / \mathrm{dl}$. Arterial blood gases included a pH 7.24, $\mathrm{PaCO}_{2} 62 \mathrm{mmHg}$, and a $\mathrm{PaO}_{2}$ $79 \mathrm{mmHg}$ on $\mathrm{FiO}_{2} 100 \%$. Oral intubation failed, and an emergency cricothyrotomy was done. She did not receive corticosteroids or scorpion antivenom.

The patient was transferred to University Medical Center in Lubbock, TX, using mechanical ventilation during transport. Her husband reported that she had allergies to aspirin, penicillins, doxycycline, nabumetone, and bee stings. Vital signs included blood pressure $89 / 63 \mathrm{mmHg}$, heart rate 90 beats per minute, and respiratory rate 16 breaths per minute. Her oxygen saturation was $96 \%$ on a $\mathrm{FiO}_{2}$ of $45 \%$. Initial 
laboratory testing showed WBC $15.3 \mathrm{k} / \mu \mathrm{L}$ with $0 \%$ eosinophils, hemoglobin $12.4 \mathrm{gm} / \mathrm{dL}$, and platelet count $225 \mathrm{k} / \mu \mathrm{L}$. A complete chemistry panel included sodium $138 \mathrm{mEq} / \mathrm{L}$, potassium $5.7 \mathrm{mEq} / \mathrm{L}$, chloride $100 \mathrm{mEq} / \mathrm{L}$, BUN $15 \mathrm{mg} / \mathrm{dL}$, and creatinine $0.9 \mathrm{mg} / \mathrm{dl}$. The patient received one dose of intramuscular epinephrine $(0.3 \mathrm{mg})$, three days of norepinephrine by continuous infusion, and methylprednisolone followed by prednisone throughout the hospitalization. She also received famotidine for four days, diphenhydramine for two days, and albuterol-ipratropium for nine days. She required mechanical ventilation for eight days. ECG revealed episodic sinus bradycardia. Her echocardiogram was normal except for mild tricuspid regurgitation and an estimated right ventricular systolic pressure $43 \mathrm{mmHg}$ (normal: $<30 \mathrm{mmHg}$ ). She did not develop pulmonary edema, acute kidney injury, or any neurologic complications. The ENT service was consulted, and the patient underwent surgery to place a tracheostomy tube. The patient was eventually placed on a tracheostomy collar and discharged. She did not receive scorpion antivenom.

\section{Discussion}

Scorpion stings occur worldwide with the greatest incidences in subtropical and tropical regions. An epidemiologic study published in Wilderness and Environmental Medicine found that the clinical presentations depended on the species of scorpion and that severe complications included pulmonary edema, acute respiratory failure, cardiac arrhythmias, myocarditis, hypertension, hypotension, coma, seizures, and multiorgan failure. The study concluded that treatment generally includes three components: symptomatic measures, vital function support, and the administration of antivenom. The study also found that the patient prognoses were heavily dependent on the amount of time elapsed from the sting to the beginning of treatment. ${ }^{2}$

Scorpion venom contains numerous chemicals, including peptides, enzymes, mucoproteins, free amino acids, nucleotides, amines, lipids, heterocyclic components, inorganic salts, and other compounds. ${ }^{3}$ The toxic components of scorpion venom are small peptides that target ion channels found in both mammals and insects. The toxins that are the most clinically significant to mammals are the alpha-toxins which block voltage-gated sodium channels, leading to a prolonged depolarization and neuronal excitation. This stimulates both sympathetic and parasympathetic autonomic centers and releases epinephrine, norepinephrine, neuropeptide $Y$ and endothelin-1. ${ }^{4}$ The parasympathetic effects of the venom are generally less severe than the sympathetic responses. Signs of parasympathetic involvement appear soon after the sting happens, and the patient may have bronchospasm, bronchorrhea, salivation, miosis, diarrhea, bradycardia, and diaphoresis. ${ }^{4}$ The sympathetic effects are often much more severe because of the additional effects of circulating catecholamines. Patients may develop myocardial injury, pulmonary edema, and cardiogenic shock. The blood pressure initially rises with an increase in cardiac output and then falls if there is a decrease in left ventricular function. Unlike the parasympathetic effects which appear quickly and subside, the sympathetic activity can persist for over 18 hours because of the release of catecholamines. ${ }^{4,5}$ Central nervous system effects are uncommon because the toxins cross the bloodbrain barrier poorly. However, the venom in scorpions found in North America can cause severe neurologic complications, especially in children. ${ }^{6}$ These include severe agitation with uncoordinated arm flailing, ocular motor and visual abnormalities, and respiratory abnormalities, including increased secretions, airway obstruction, abnormal ventilatory control, and occasionally noncardiogenic pulmonary edema. The neurological complications may be due to hypertensive encephalopathy and catecholamine release. Only about $10 \%$ of stings result in severe systemic envenomation; most of the time the sting causes erythema, edema, and paresthesias around the site of the sting. ${ }^{4}$

In healthy adults, scorpion stings rarely cause a medical emergency. Anaphylaxis from a sting is possible if the patient has been stung before or if there is a cross reaction to components in the venom related to prior insect stings. Nugent et al studied the cross reactivity of scorpion venom with imported fire ant venom and found significant cross-reactivity 


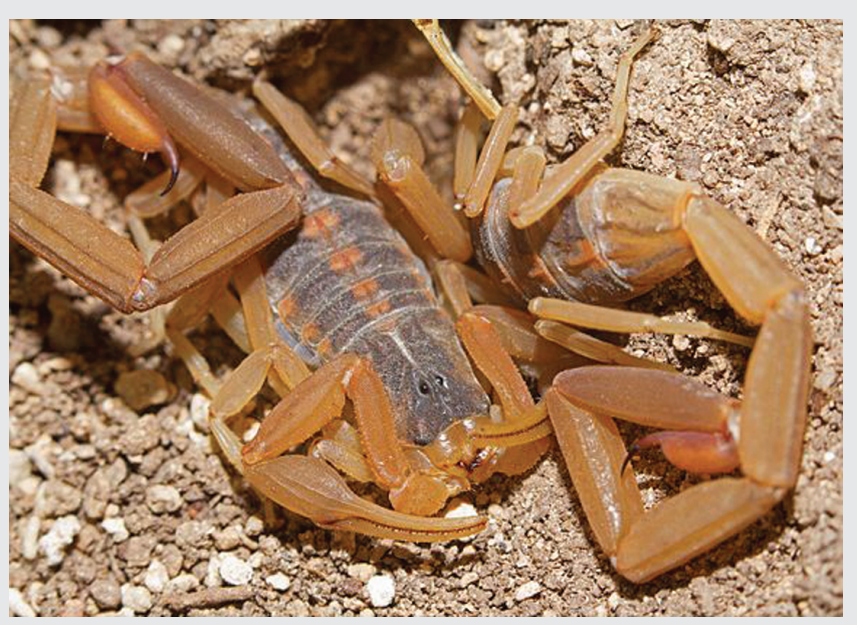

Figure. Striped bark scorpion (Centruroides vittatus) https://commons.wikimedia.org/wiki/ Category:Centruroides_vittatus\#/media/File:Striped_ Bark_Scorpion_(Buthidae,_Centruroides_vittatus)_ (26294838801).jpg. Accessed 10-23-2017

between these two venoms. Consequently, people with systemic reaction to imported fire ants are at a 20 fold higher risk for allergic reactions to scorpion venom. ${ }^{7}$ In addition, scorpion venoms have strong cross-reactivity with bee venom. ${ }^{8}$ The World Allergy Organization estimates that $1 \%$ to $7 \%$ of the general population have allergic reactions to insect stings. ${ }^{9}$ The CDC reports as many as 100 people in the United States die every year as a result of allergic reaction to insect stings. ${ }^{10}$ This number could be underreported because these deaths may be attributed to heart attacks, sunstrokes, or other causes. We think that our patient had an acute anaphylactic reaction to scorpion venom resulting in mucosal edema and upper airway obstruction. This reaction was most likely secondary to cross reactivity between scorpion venom and bee venom to which she had an allergy. The patient's prolonged requirement for mechanical ventilation following tracheostomy was probably explained, in part, by her underlying sleep apnea syndrome complicated by pulmonary hypertension. The patient did not appear to have an associated toxic reaction due to the lack of rapid swelling at the sting site, nausea, diarrhea, lightheadedness, and muscle spasms.
Antivenom can be used in patients with scorpion stings; it binds to toxins so that they are unable to reach target sites. Its use has been controversial, and its low cost effectiveness typically limits its use to cases with severe systemic envenomation. ${ }^{11}$ Two studies showed that scorpion sting victims who received antivenom in addition to prazosin recovered much faster than those who received only prazosin. ${ }^{12,13}$ Unfortunately, by the time the patients have developed a severe envenomation reaction, the antivenom may not be very effective because it cannot reverse the injury already induced by the toxins. ${ }^{3}$ The antivenom needs to bind toxins before they are able to exert their effect and release catecholamines. Antivenom is a protein, so the risk of anaphylaxis is always present. Anaphylactic reactions to antivenom are due to either complement activation by $\mathrm{lgG} /$ residual $\mathrm{FC}$ fragments or direct stimulation of mast cells. ${ }^{14}$ Within two hours the patient will develop itching, urticaria, dry cough, fever, nausea, vomiting, diarrhea, tachycardia, and abdominal colic. ${ }^{14} \mathrm{~A}$ minority of these patients may experience more severe symptoms, such as hypotension, bronchospasm, and angioedema. Some patients develop a delayed reaction following antivenom administration. ${ }^{15}$ There are no absolute contraindications to antivenom, but patients who have had reactions to horse or sheep serum in the past or have a history of atopic diseases are at a higher risk for severe reactions. ${ }^{14}$ Epinephrine is the treatment of choice in the event of an anaphylactic reaction, and promethazine has shown the most efficacy for prophylaxis. ${ }^{14}$ Serum sickness developed in $6 \%$ of patients receiving scorpion antivenom but they responded quickly to oral corticosteroids and/or antihistamines. ${ }^{15}$

In summary, scorpion stings can cause a range of reactions which includes severe systemic envenomation, resulting in respiratory failure, hemodynamic instability, and severe neurologic complications. These occur more frequently in children and depend on the scorpion species. In addition, some patients can have acute anaphylactic reactions to scorpion venom secondary to cross-reactivity from venoms from bees and ants. The initial management may require cardiorespiratory resuscitation and antivenom, if available. In adults, the clinical course is also influenced by underlying comorbidity. 
Article citation: Castillo A, Attaluri P. Acute respiratory failure following scorpion stings: Anaphylaxis or severe systemic envenomation? The Southwest Respiratory and Critical Care Chronicles 2018;6(22):47-50.

From: Department of Internal Medicine, Texas Tech University Health Sciences Center, Lubbock, Texas

Submitted: $10 / 25 / 2017$

Accepted: $1 / 5 / 2018$

Reviewers: James A Tarbox MD

Conflicts of interest: none

\section{REFERENCES}

1. Mowry JB, Spyker DA, Brooks DE, et al. 2015 Annual Report of the American Association of Poison Control Centers' National Poison Data System (NPDS): $33^{\text {rd }}$ Annual Report, Clinical Toxicology 2016; 54:924-1109.

2. Santos MSV, Silva CGL, Neto BS. Clinical and epidemiological aspects of scorpionism in the world: a systematic review. Wilderness and Environmental Medicine 2016; 27 (4):504-518.

3. Ortiz E, Gurrola GB, Schwartz E, et al. Scorpion venom components as potential candidates for drug development. Toxicon 2015;93:125-135.

4. Isbister GK, Bawaskar HS. Scorpion envenomation. New Engl J Med 2014; 371(5):457-63.

5. Pandi K, Krishnamurthy S, Srinivasaraghavan R, et al. Efficacy of scorpion antivenom plus prazosin versus prazosin alone for Mesobuthus tamulus scorpion sting envenomation in children: a randomized controlled trial. Arch Dis Child 2014;99:575-80.

6. Boyer LV, Theodorou AA, Berg RA, et al. Antivenom for critically ill children with neurotoxicity from scorpion stings. N Engl J Med 2009; 360(20):2090-8.

7. Nugent JS, More DR, Hagan LL, et al. Cross-reactivity between allergens in the venom of the common striped scorpion and the imported fire ant. J Allergy Clin Immunol 2004; 114:383-386.

8. Lipps, BV, Khan, AA. Antigenic cross reactivity among the venoms and toxins from unrelated diverse sources. Toxicon 2000; 38(7):973-80.

9. Insects and scorpions. Centers for Disease Control and Prevention, Centers for Disease Control and Prevention, https://www.cdc.gov/niosh/topics/insects/default.html, 1 July 2016. Accessed 11-10-2017.

10. Heddle R, Golden D. Allergy to insect stings and bites. WAO, August 2015, www.worldallergy.org/professional/allergic _ diseases_center/insect_allergy/. Accessed 24 Sept. 2017.

11. Armstrong EP, Bakall M, Skrepnek GH, et al. LV. Is scorpion antivenom cost effective as marketed in the United States? Toxicon 2013; 76:394-8.

12. Bawaskar HS, Bawaskar PH. Efficacy and safety of scorpion antivenom plus prazosin compared with prazosin alone for venomous scorpion (Mesobuthus tamulus) sting: randomized open label clinical trial. BMJ 2011; 342:c7136.

13. Warrell DA. Guidelines for the management of snakebites. Antivenom reactions. apps.searo.who.int/PDS_DOCS/ B4508.pdf.

14. LoVecchio F, Welch S, Klemens J, et al. Incidence of immediate and delayed hypersensitivity to Centruroides antivenom. Ann Emerg Med 1999; 34:615-619.

15. Sanaei-Zadeh H. Hypersensitivity reaction to scorpion antivenom. Indian Dermatol Online J 2014; Suppl S1:51-2. 